\title{
Surge in Anaplasmosis Cases in Maine, USA, 2013-2017
}

\author{
Susan P. Elias, Jessica Bonthius, Sara Robinson, Rebecca M. Robich,
} Charles B. Lubelczyk, Robert P. Smith, Jr.

Incidence of human granulocytic anaplasmosis is rising in Maine, USA. This increase may be explained in part by adoption of tick panels as a frequent diagnostic test in persons with febrile illness and in part by range expansion of Ixodes scapularis ticks and zoonotic amplification of Anaplasma phagocytophilum.

L yme disease is the most common vectorborne $\checkmark$ disease in Maine. Borrelia burgdorferi, the agent of Lyme disease, is transmitted through the bite of infected blacklegged ticks (Ixodes scapularis). Lyme disease cases in Maine increased from a single case in 1986 to 1,844 in 2017, reflecting the northward range expansion of I. scapularis ticks (1). Anaplasma phagocytophilum, the cause of human granulocytic anaplasmosis (HGA), is also transmitted by I. scapularis ticks and is the second most common tickborne illness in Maine. Only 45 HGA cases were reported during 2000-2008 (2), but case reports rose dramatically during 2013-2017, generating media attention $(3,4)$. The Maine Center for Disease Control and Prevention (MECDC) reported 663 cases of anaplasmosis in 2017, a $605 \%$ increase from 94 cases in 2013, in contrast with Lyme disease cases, which increased by only $33 \%$ (1,384 in 2013 to 1,844 in 2017) (5).

We sought to determine whether the increase in anaplasmosis cases reflected broader geographic transmission of $A$. phagocytophilum from ticks to humans through range expansion of I. scapularis ticks, increased testing effort through increased use of tick panels that detect multiple pathogens by PCR, or both. Evidence for increased transmission would include geographic range expansion of

Author affiliations: Maine Medical Center Research Institute, Scarborough, Maine, USA (S.P. Elias, R.M. Robich,

C.B. Lubelczyk, R.P. Smith, Jr.); University of Southern Maine, Portland, Maine, USA (J. Bonthius); Maine Center for Disease Control and Prevention, Augusta, Maine, USA (S. Robinson)

DOI: https://doi.org/10.3201/eid2602.190529
HGA incidence and hospitalizations. Evidence of increased testing effort would be increased use of tick panels, which could lead to discovery of mild A. phagocytophilum infections, especially pediatric cases, because HGA in children is generally a mild illness (6).

\section{The Study}

MECDC provided the number of confirmed and probable cases for Maine residents during 2008-2017, available by county of residence and age of onset. For 2013-2017, we obtained the annual number of hospitalizations for Lyme disease and HGA for Maine residents, with age and county of residence at admission, from the Maine Health Data Organization.

We obtained the annual number of multipathogen (including HGA) PCR tick panel orders during 2013-2017 from NorDx and Mayo Medical Laboratories (MML). NorDx (Scarborough, ME, USA) started using its panel for the agents of HGA and babesiosis in 2015 (H. Webber, NorDx, pers. comm., 2018 Sep 12). All orders were for Maine patients (travel history not specified). MML provided data from 2 branch laboratories, Mayo Clinic Rochester (MCR; Rochester, MN, USA) and Mayo Medical Laboratories, New England (MMLNE; Andover, MA, USA). MML has offered the panel for $\approx 10$ years; the panel contains a PCR test for the agents of human monocytic ehrlichiosis, HGA, babesiosis, and Borrelia miyamotoi infections (B. Pritt, MML, pers. comm., 2018 Aug 3). MML data comprised specimens sent to MML from Maine clients, without patient residence or travel history (B. Pritt, A. Boerger, MML, pers. comm., 2018 Oct 8). We were unable to obtain data from other laboratories; however, MML and NorDx combined accounted for $72 \%$ of HGA test reports sent to MECDC during 2013-2017. The MML panel had sensitivity and specificity of 1 for detection of A. phagocytophilum compared with standard PCR (7). The NorDx panel had sensitivity and specificity of 1 compared 
Table 1. Number of HGA and Lyme disease cases and incidence, Maine, USA, 2008-2017*

\begin{tabular}{|c|c|c|c|c|c|c|c|c|c|c|}
\hline \multirow[b]{3}{*}{ Year } & \multicolumn{8}{|c|}{ HGA } & \multirow{2}{*}{\multicolumn{2}{|c|}{ Lyme }} \\
\hline & \multicolumn{4}{|c|}{ Cases } & \multicolumn{4}{|c|}{ Incidence } & & \\
\hline & All ages & $0-17 y$ & $18-64 y$ & $\geq 65 y$ & All ages & $0-17 y$ & $18-64 y$ & $\geq 65 \mathrm{y}$ & Cases & Incidence† \\
\hline 2008 & 17 & 0 & 11 & 6 & 1.3 & 0 & 1.3 & 3.0 & 909 & 68.3 \\
\hline 2009 & 15 & 0 & 9 & 6 & 1.1 & 0 & 1.1 & 2.9 & 976 & 73.4 \\
\hline 2010 & 17 & 1 & 10 & 6 & 1.3 & 0.4 & 1.2 & 2.8 & 752 & 56.6 \\
\hline 2011 & 26 & 0 & 12 & 14 & 2.0 & 0 & 1.4 & 6.5 & 1013 & 76.3 \\
\hline 2012 & 52 & 0 & 37 & 15 & 3.9 & 0 & 4.4 & 6.6 & 1113 & 83.7 \\
\hline 2013 & 94 & 0 & 56 & 38 & 7.1 & 0 & 6.7 & 16.2 & 1384 & 104.2 \\
\hline 2014 & 191 & 10 & 102 & 79 & 14.4 & 3.9 & 12.3 & 32.5 & 1411 & 106.1 \\
\hline 2015 & 185 & 9 & 92 & 84 & 13.9 & 3.5 & 11.2 & 33.6 & 1215 & 91.4 \\
\hline 2016 & 372 & 9 & 206 & 157 & 27.9 & 3.5 & 25.2 & 60.8 & 1497 & 112.4 \\
\hline 2017 & 663 & 13 & 304 & 346 & 49.7 & 5.1 & 37.3 & 129.6 & 1844 & 138.5 \\
\hline Change 2013-2017 & $605 \%$ & & $443 \%$ & $811 \%$ & $602 \%$ & & $454 \%$ & $701 \%$ & $33 \%$ & $33 \%$ \\
\hline
\end{tabular}

with panels of MML and other laboratories (H. Webber, pers. comm., 2019 Aug 29).

The HGA incidence rate, hospitalization rate, complications, and death rate increase with age (8), whereas Lyme incidence has a bimodal distribution, with peaks in young children and older adults (9). We tabulated HGA cases and incidence for 2008-2017 overall and by age class and tabulated HGA hospitalizations 2013-2017 overall and by age class and annual laboratory testing effort. For comparison, we included annual overall Lyme incidence and hospitalizations. We compared percentage changes from 2013 to 2017 in disease incidence and hospitalizations. To visualize geographic expansion of HGA, we plotted side-byside maps of county-level incidence and populationadjusted hospitalization rates for 2013 versus 2017.

During 2013-2017, a total of 1,505 anaplasmosis cases were reported $(10)$. Of these, $85.6 \%(1,289)$ were confirmed $(1,286$ by PCR and 3 by 4 -fold antibody titer increase) and $14.4 \%$ (216) probable (203 with a single titer result, 8 with $<4$-fold titer increase, 5 with morulae visualization). Statewide, anaplasmosis incidence rose from 7 cases/100,000 persons in 2013 to 50 cases/100,000 persons in 2017, a 602\% increase, compared with a $33 \%$ increase for Lyme disease incidence (Table 1). Hospitalizations for HGA rose from 36 in 2013 to 119 in 2017, a 231\% increase, compared with a $27 \%$ decline in hospitalizations for Lyme disease (Table 2). Combined tick panel use by MML and NorDx rose from 773 in 2013 to 9,157 in 2017, a $1,085 \%$ increase (Table 2).

Among 39 pediatric HGA cases, 1 occurred in 2010 and the remaining 38 during 2014-2017, representing $1.7 \%-5.2 \%$ of total cases per year during 2014-2017 (Table 1). Even though hospitalizations increased for persons $18-64$ and $\geq 65$, there were no hospitalizations for children.

Anaplasmosis incidence and hospitalizations underwent geographic range expansion during 2013-2017 (Figures 1, 2). Anaplasmosis incidence was highest in Lincoln and Knox Counties, in Maine's midcoast region, where incidence ranged from 29 cases/100,000 persons in 2013 to 278 cases/100,000 persons in 2017 (Figure 1, panels A, B).

\section{Conclusions}

We conclude that the surge in anaplasmosis incidence in Maine, an increase of 602\% from 2013 to 2017, was a combination of increased transmission and testing effort, although we cannot partition the relative

Table 2. Number of hospitalizations for HGA and Lyme disease and number of PCR-based tickborne disease panels, Maine, USA, 2013-2017*

\begin{tabular}{|c|c|c|c|c|c|c|c|c|c|c|}
\hline \multirow[b]{3}{*}{ Year } & \multicolumn{5}{|c|}{ Hospitalizations } & \multirow{2}{*}{\multicolumn{5}{|c|}{ Tick panels $†$}} \\
\hline & \multicolumn{4}{|c|}{ HGA } & \multirow{2}{*}{$\begin{array}{l}\text { Lyme, } \\
\text { all ages }\end{array}$} & & & & & \\
\hline & All ages & $0-17 y$ & $18-64 y$ & $>65 y$ & & MML-R & MML-NE & Total MML & NorDx & All \\
\hline 2013 & 36 & 0 & 9 & 27 & 66 & 0 & 773 & 773 & 0 & 773 \\
\hline 2014 & 75 & 0 & 25 & 50 & 55 & 0 & 1,479 & 1,479 & 0 & 1,479 \\
\hline 2015 & 68 & 0 & 19 & 49 & 45 & 0 & 1,066 & 1,066 & 875 & 1,941 \\
\hline 2016 & 123 & 0 & 42 & 81 & 47 & 596 & 122 & 718 & 5,259 & 5,977 \\
\hline 2017 & 119 & 0 & 24 & 95 & 48 & 973 & 0 & 973 & 8,184 & 9,157 \\
\hline Change 2013-2017 & $+231 \%$ & & $+167 \%$ & $+252 \%$ & $-27 \%$ & & & & & $+1,085^{\circ}$ \\
\hline
\end{tabular}

*Hospitalizations are Maine hospital inpatient encounters, provided through the Maine Health Data Organization. HGA, human granulocytic anaplasmosis; MML, Mayo Medical Laboratories.

†Tick panels performed at Mayo Clinic Rochester, Rochester, MN, USA (MML-R); MML, New England, Andover, MA, USA (MML-NE, closed in 2016); and NorDx, Scarborough, ME, USA. 

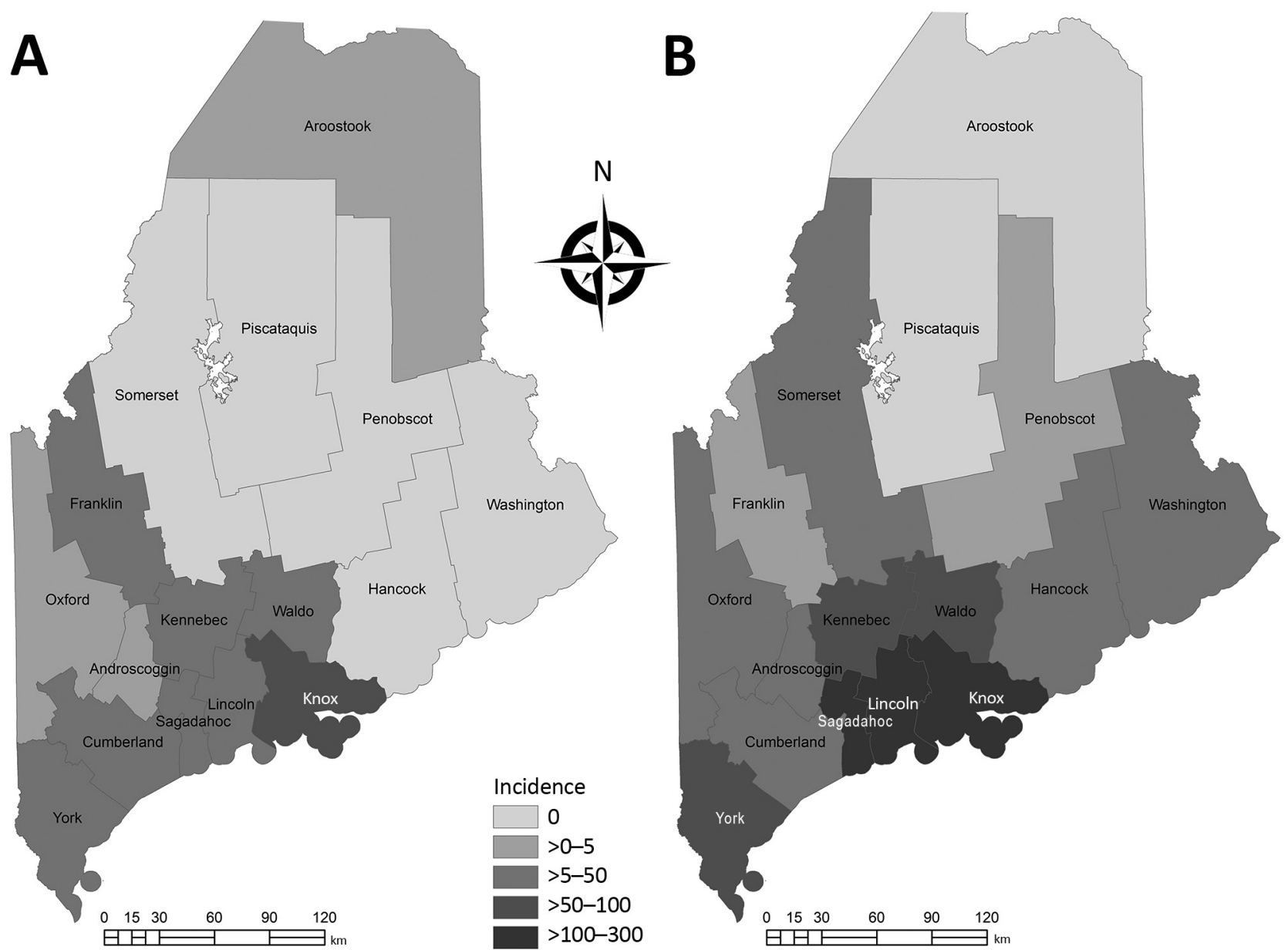

Figure 1. Human granulocytic anaplasmosis incidence (cases/100,000 persons), Maine, USA, 2013 (A) and 2017 (B). Statewide incidence increased 602\% during 2013-2017.

contribution of each. The $231 \%$ rise in hospitalized patients with HGA and the geographic expansion of HGA incidence and hospitalization indicate increased transmission. Range expansion of I. scapularis ticks in Maine likely has contributed to the rise in HGA cases in areas where this tick species is emergent (i.e., a recent colonizer). In addition, zoonotic amplification of A. phagocytophilum is likely occurring where I. scapularis ticks are established. Because of less efficient enzootic transmission, human infection with Babesia microti, the agent of babesiosis, lags behind B. burgdorferi transmission over time and space (11). Less efficient enzootic transmission of $A$. phagocytophilum also may be the case, but we know of no confirmatory studies.

Concurrent to increased transmission was the $1,085 \%$ increase in tickborne disease panel testing performed by the 2 major providers of testing results to Maine during 2013-2017. Increased testing effort may reflect increased clinician and patient awareness and ready availability of tickborne disease panels that detect multiple pathogens. These panels may lead to detection of mild A. phagocytophilum infections or coinfections in persons with nonspecific febrile illness, as suggested by increased detection of less severely ill persons, such as children. Thirty-eight of 39 pediatric HGA cases were reported after 2013, but there were no pediatric hospitalizations. Before the use of panels, pediatric HGA cases may have been ascribed to another illness with similar symptoms.

Studies relying on diagnostic tests are subject to test sensitivity and specificity. PCR is the most effective diagnostic test during early-stage $A$. phagocytophilum infection with high sensitivity and specificity $(12,13)$. In this study, false positive PCR results were unlikely, based on test specificities reported by Mayo and NorDx.

Collaboration among all state health departments and testing laboratories across New England could help extend our findings. Vermont cases increased 1,078\%, from 37 in 2013 to 399 in 2017 (14), and New 


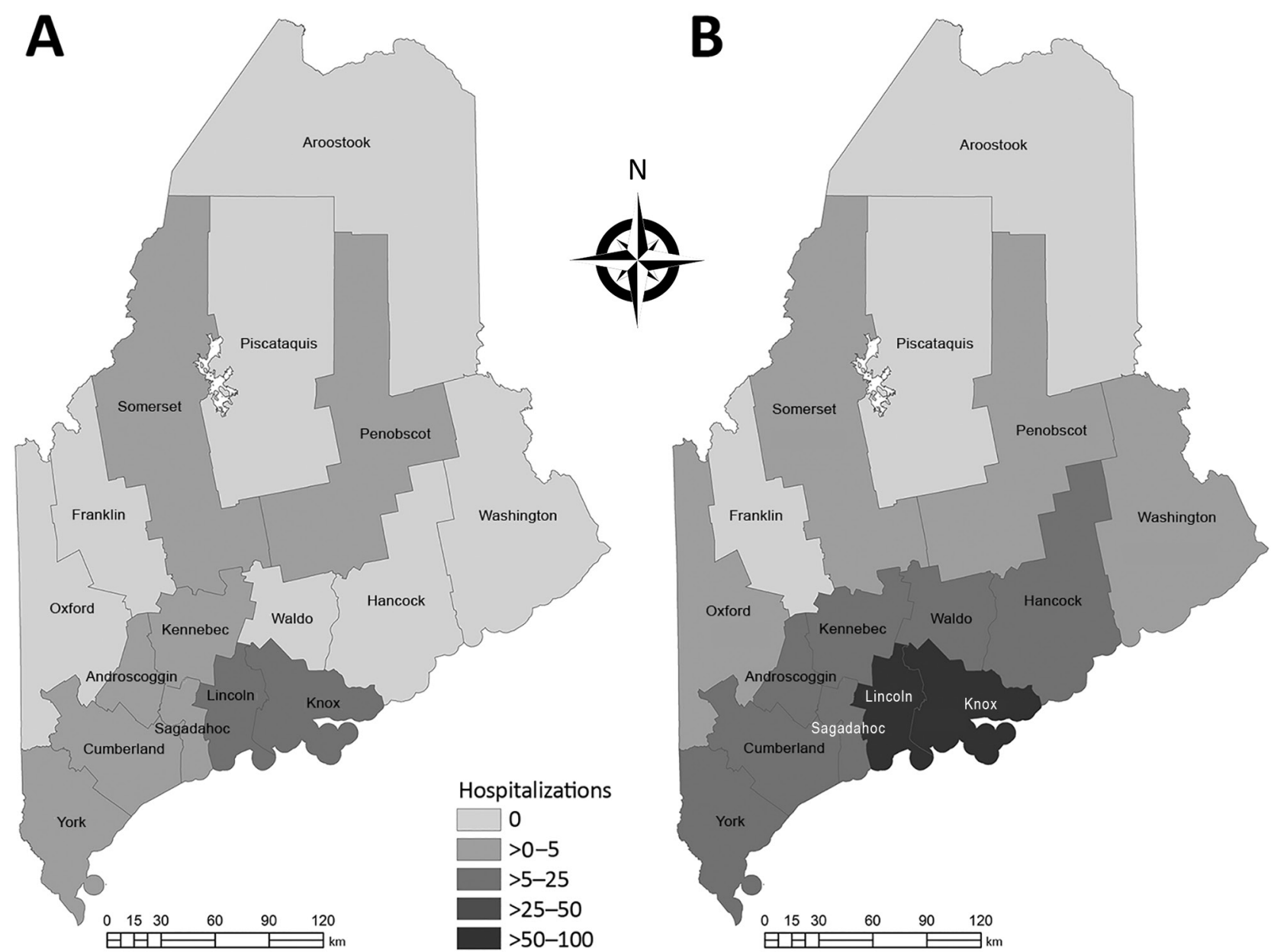

Figure 2. Hospitalizations (per 100,000 persons) for human granulocytic anaplasmosis, Maine, USA, 2013 (A) and 2017 (B). Statewide hospitalizations increased $231 \%$ during $2013-2017$.

Hampshire cases increased $260 \%$, from 88 in 2013 to 317 in 2017 (15). Correlation between incidence and testing effort at the county level would corroborate a relationship between rising tickborne diseases and testing effort, if panel data included patient county of residence and travel history. Corroborating datasets on density of A. phagocytophilum-infected I. scapularis ticks would also help clarify the risks posed to human health.

\section{Acknowledgments}

For NorDx data, we thank Monica Ianosi-Irimie, Haley Webber, and Lisa Webster. For Mayo Medical Laboratories data, we thank Bobbi Pritt and Aimee Boerger. For hospitalization data, we thank the Maine Health Data Organization, as well as Hilary Perrey and Lori Travis.

Partial salary support for co-authors J.B. and S.R. came from the Centers for Disease Control and Prevention grants Building Resilience Against Climate Effects (BRACE) and Epidemiology and Laboratory Capacity (ELC).

\section{About the Author}

Dr. Elias is a staff scientist at the Maine Medical Center Research Institute, Lyme and Vector-borne Disease Laboratory, Scarborough, Maine, USA. Her primary research interests include epidemiology and ecology of emergent vectorborne diseases.

\section{References}

1. Rand PW, Lacombe EH, Dearborn R, Cahill B, Elias S, Lubelczyk CB, et al. Passive surveillance in Maine, an area emergent for tick-borne diseases. J Med Entomol. 2007;44:1118-29. https:// doi.org/10.1093/jmedent/ 44.6.1118

2. Cahill B, Lubelczyk C, Smith R, Gensheimer K, Robbins A, Robinson S, et al.; Centers for Disease Control and Prevention (CDC). Anaplasmosis and ehrlichiosis - Maine, 2008. MMWR Morb Mortal Wkly Rep. 2009;58:1033-6.

3. Wight P. Tick-borne anaplasmosis on the rise in Maine. 2017 [cited 2019 May 9]. https:/ / bangordailynews. com/2017/11/13/health/tick-borne-anaplasmosison-the-rise-in-maine 
4. Lawlor J. Tick-borne anaplasmosis surging in Maine-and it's worse than Lyme. 2017 [cited 2019 May 9]. https:/ /www. pressherald.com/2017/11/13/anaplasmosis-cases-surgingin-maine

5. Maine Center for Disease Control. Reportable infectious diseases in Maine-2017 summary. 2018 [cited 2018 Sep 20]. https://www.maine.gov/dhhs/mecdc/infectious-disease/ epi/publications/\#annualreports

6. Sigurjonsdottir VK, Feder HM Jr, Wormser GP. Anaplasmosis in pediatric patients: case report and review. Diagn Microbiol Infect Dis. 2017;89:230-4. https:/ / doi.org/ 10.1016/j.diagmicrobio.2017.08.003

7. Mayo Clinic Laboratories. Test ID: EHRL. Ehrlichia/ anaplasma, molecular detection, PCR, blood [cited 2019 Sep 29]. https:/ / www.mayocliniclabs.com/test-catalog/ Clinical+and+Interpretive/84319

8. Dahlgren FS, Heitman KN, Drexler NA, Massung RF, Behravesh CB. Human granulocytic anaplasmosis in the United States from 2008 to 2012: a summary of national surveillance data. Am J Trop Med Hyg. 2015;93:66-72. https:// doi.org/10.4269/ajtmh.15-0122

9. Schwartz AM, Hinckley AF, Mead PS, Hook SA, Kugeler KJ. Surveillance for Lyme disease - United States, 2008-2015. MMWR Surveill Summ. 2017;66(No. SS-22):1-12. https://doi.org/10.15585/mmwr.ss6622a1

10. U.S. Centers for Disease Control and Prevention. Ehrlichiosis and anaplasmosis 2008 case definition [cited 2019 Aug 29]. https://wwwn.cdc.gov/nndss/conditions/anaplasmaphagocytophilum-infection/case-definition/2008/
11. Diuk-Wasser MA, Vannier E, Krause PJ. Coinfection by Ixodes tick-borne pathogens: ecological, epidemiological, and clinical consequences. Trends Parasitol. 2016;32:30-42. https://doi.org/10.1016/j.pt.2015.09.008

12. Hansmann $Y$, Jaulhac B, Kieffer $P$, Martinot M, Wurtz E, Dukic R, et al. Value of PCR, serology, and blood smears for human granulocytic anaplasmosis diagnosis, France. Emerg Infect Dis. 2019;25:996-8. https:/ / doi.org/10.3201/ eid2505.171751

13. Schotthoefer AM, Meece JK, Ivacic LC, Bertz PD, Zhang K, Weiler $\mathrm{T}$, et al. Comparison of a real-time PCR method with serology and blood smear analysis for diagnosis of human anaplasmosis: importance of infection time course for optimal test utilization. J Clin Microbiol. 2013;51:2147-53. https://doi.org/10.1128/JCM.00347-13

14. Vermont Department of Health. Anaplasmosis [cited 2018 Sep 25]. https:// www.healthvermont.gov/disease-control/ tickborne-diseases/anaplasmosis

15. New Hampshire Department of Health and Human Services. Tickborne diseases in New Hampshire [cited 2018 Sep 25]. https:/ / www.hanovernh.org/sites/hanovernh/ files/uploads/20180518_tickborne_disease_han_with_ attachments.pdf

Address for correspondence: Susan P. Elias, Maine Medical Center Research Institute, Lyme and Vector-borne Disease Laboratory, 81 Research Dr, Scarborough, ME 04074, USA; email: susan.elias@maine.edu

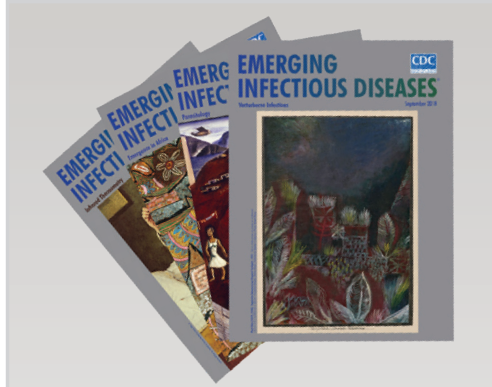

- Ethics of Infection Control Measures for Carriers of Antimicrobial Drug-Resistant Organisms

- National Surveillance for Clostridioides difficile Infection, Sweden, 2009-2016

- Travel-Associated Zika Cases and Threat of Local Transmission during Global Outbreak, California, USA

- Distinguishing Japanese Spotted Fever and Scrub Typhus, Central Japan, 2004-2015

- Event-Based Surveillance at Community and Healthcare Facilities, Vietnam, 2016-2017

- Case Report and Genetic Sequence Analysis of Candidatus Borrelia kalaharica, Southern Africa

- Novel Orthopoxvirus and Lethal Disease in Cat, Italy

- Emergence of Carbapenemase-Producing Enterobacteriaceae, South-Central Ontario, Canada

September 2018

\section{Vectorborne Infections}

- From Culturomics to Clinical Microbiology and Forward

- Association of Batai Virus Infection and Encephalitis in Harbor Seals, Germany, 2016

- Use of Favipiravir to Treat Lassa Virus Infection in Macaques

- Aortic Endograft Infection with Mycobacterium chimaera and Granulicatella adiacens, Switzerland, 2014

- Estimating Frequency of Probable Autochthonous Cases of Dengue, Japan

- Correlation of Severity of Human Tick-borne Encephalitis Virus Disease and Pathogenicity in Mice

- Increasing Prevalence of Borrelia burgdorferi sensu strictoInfected Blacklegged Ticks in Tennessee Valley, Tennessee, USA

- Susceptibility of White-Tailed Deer to Rift Valley Fever Virus

- Outbreak of Pneumococcal Meningitis, Paoua Subprefecture, Central African Republic, 2016-2017 